\title{
Electron Beam Printed Hydrogels as a Hydration Source for Graphene Encapsulated Specimens
}

Chris Arble and Andrei Kolmakov

NIST, United States

In-situ optical, electron, and X-ray spectromicroscopies have been proven to be powerful tools to study liquid samples and interfaces relevant to electrochemistry, environmental studies, and biomedical fields. Encapsulation of the hydrated samples with molecularly impermeable photon and electron transparent membranes1, such as graphene2, 3, is a simple approach to isolate the sample from the vacuum or dry environment. The amount of trapped liquid around the specimen, however, is minuscule and the hydration retention time, therefore, is small in a vacuum and strongly depends on the quality of the encapsulating graphene and its adhesion to the substrate. Besides, irradiation of the microscopic volumes of water with ionizing radiation leads to a fast buildup of radiolitic products what drastically alters physicochemical processes in sensitive samples such as living cells.

Herein we report on the development of a hydrogel-based technology that drastically prolongs the hydration state of the graphene encapsulated objects. Figure 1 depicts the principle of this method.

A special hydrogel patterning procedure was developed to immobilize live cells on SiN $50 \mathrm{~nm}$ thick membranes with micron-scale precision4. For this, a mixture of the biocompatible 20\% w/v PEGDA solution in PBS and cells was drop cast onto the front side of the SiN membrane, sealed in a special chamber, and cross-linked with an electron beam from the backside through the SiN window (Figure 1a). The cells of interest, therefore, become immobilized by the crosslinked hydrogel the shape, thickness (and porosity) of which can be tuned by varying the energy and dose of the irradiation. The water retention time inside such an encapsulated specimen was measured in a vacuum with EDX and fluorescence microscopy using fluorescence as a hydration marker.

This method can be developed further to incorporate microprinted gel fluidic channels for feeding and supporting live cells as well as to pattern analytical probes such as electrodes to study local electrophysiology in vivo. Overall, proposed here method facilitates the study of biological samples, under physiological conditions with techniques that require dry/or vacuum conditions. 

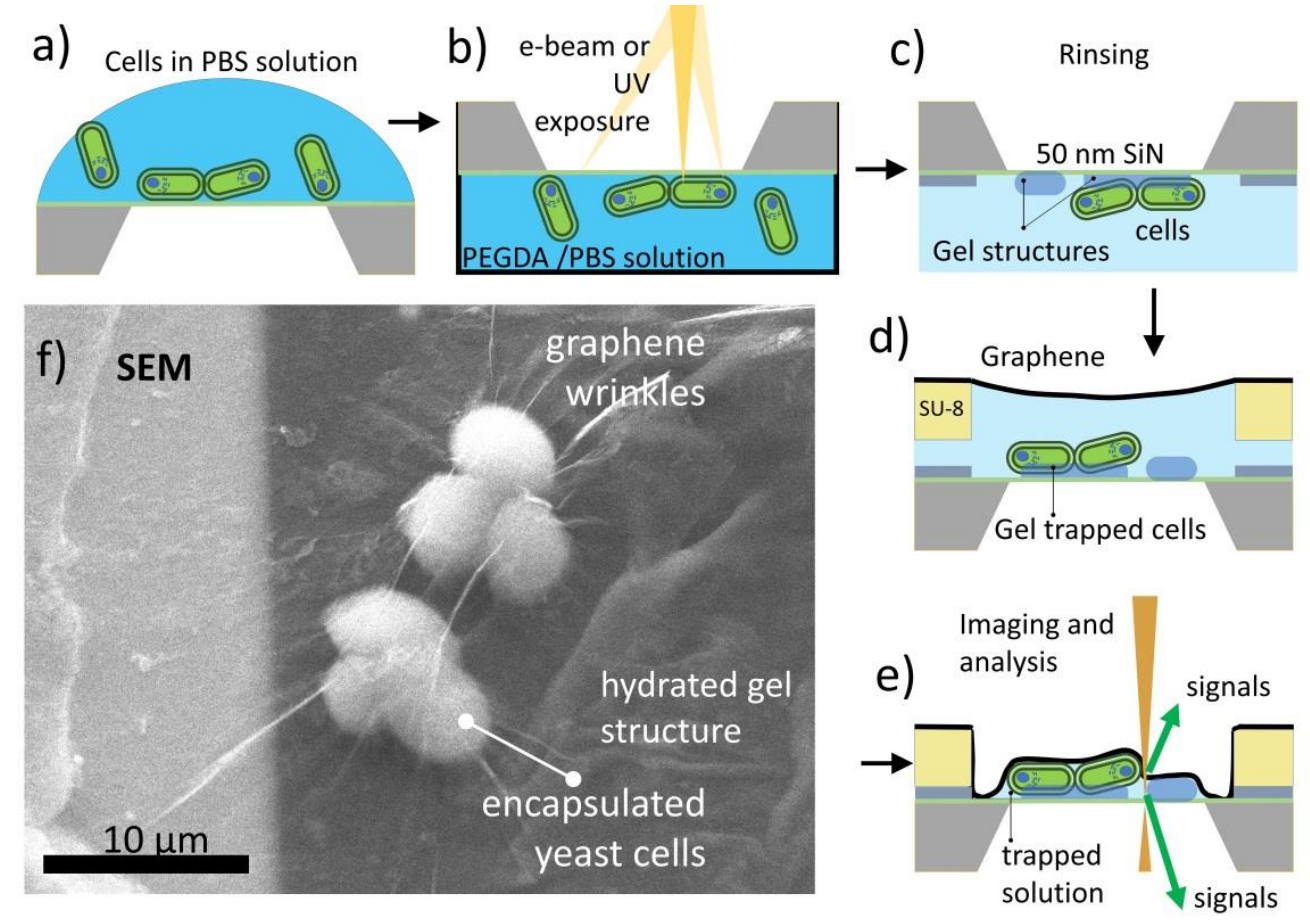

Figure 1. a)-e) Cell addressable immobilization and encapsulation with graphene encapsulation c) SEM image of encapsulated yeast cell

\section{References}

1. X. Ma, et al., Science of The Total Eenvironment, 2010, 408, 3053-3061.

2. J. D. Stoll and A. Kolmakov, Nanotechnology, 2012, 23, 505704.

3. J. Park, et al., Nano letters, 2015, 15, 4737-4744.

4. T. Gupta, et al., ACS Nano, 2020, 14, 12982-12992. 